\title{
Comparison of Teaching Basic Electrocardiographic Concepts with and without ECGSIM, an Interactive Program for Electrocardiography
}

\author{
TP Patuwo $^{1}$, GS Wagner ${ }^{2}$, OA Ajijola ${ }^{2}$ \\ ${ }^{1}$ Rice University, Houston, USA, ${ }^{2}$ Duke University Medical Center, Durham, USA
}

\begin{abstract}
The electrocardiogram (ECG) is an important diagnostic tool in the management of cardiac disease. Currently, most methods of introducing electrocardiography in medical schools rely on books, papers, and clinical observation (classical method). As more interactive software become available, it would be instructive for students to learn the ECG this way. This study compares the effectiveness of teaching the basics of electrocardiography using the classical method versus an interactive program, ECGSIM. Thirty-five students were randomized into two groups (classical and ECGSIM) and were taught the basic concepts of the ECG. This study demonstrates that teaching electrocardiography using ECGSIM is superior to traditional methods alone, indicating a potentially large role for interactive programs in teaching electrocardiography.
\end{abstract}

\section{Introduction}

Electrocardiographic concepts and the use of the electrocardiogram (ECG) in clinical diagnosis are important topics taught during medical school and beyond. The utility of the ECG in clinical decision making depends on the effectiveness with which the principles and interpretive techniques of electrocardiography are learned. It is therefore important that institutions educating future medical professionals are successful in teaching this highly useful clinical tool effectively. The method used by medical trainees learning to read ECGs will influence early comprehension. The classical approach to teaching the ECG relies on written material including books and manuscripts, didactic lessons from an experienced technician, and practice. An integrated combination of these methods is currently used by most institutions. The major limitation of this approach is that it provides little correlation between these ECG waveforms and the actual three-dimensional nature of the heart. The geometric properties of the heart the produce a normal electrocardiogram, as well as the deviations from normal, that produce pathologic tracings are not easily envisioned.
ECGSIM is a freely available interactive ECG simulation software that enables one to study the relationship between the electric activity within the heart and the resulting ECG waveforms recorded on the body surface. ECGSIM $^{[1]}$ provides intra-myocardial QRST wave-forms as well as body surface potential maps, which correspond to the recordings present on the ECG. ECGIM displays 4 panels (heart, thorax, membrane, ECG), allowing the observer to simultaneously observe the relationship between electrical vectors within the heart, and the recordings produced on the ECG. ECGSIM also stimulates the effect of local changes in timing and the manifestation of local ischemic regions on the body surface potentials. ECGSIM therefore presents a sequential progression of views from 4 different aspects of cardiac electrical activity in the frontal plane through the different panels; allowing the learner to consider a progression of anatomically sequential representations of the heart. Despite the obvious advantages that ECGSIM could provide, it is imperative to study whether this method of learning supplements, is equivalent to, or detracts from the ability of trainees to understand the ECG.

This study was designed to evaluate whether using ECGSIM would facilitate a more effective understanding of the ECG, as demonstrated by enhanced performance in the calculation of the mean frontal plane QRS. The specific hypothesis was that ECG naïve learners would achieve more accurate axis determination on a more consistent (measure of consistency) basis after being taught to calculate the mean electrical axis using the software ECGSIM, rather than the classical approach.

\section{Methods}

Study population

Teaching was performed at Duke University in Durham, North Carolina United States. The study was performed using students enrolled in a summer medical education program, who had no previous experience with ECG interpretation (age range 18-25). The number of students required was determined by estimating the expected difference in performance between the two 
groups of students and by the statistical significance required. A similar published study was used as a guide $^{[2]}$.The students participated on a voluntary basis.

\section{Software}

ECGSIM is freely available and was obtained from the official website http://www.ecgsim.org/.

\section{Study design}

All subjects received oral instruction by one of the investigators (OAA) about the basic anatomy, mechanics, and electrophysiology of the heart. An instruction manual and an oral presentation were provided for all students (New York University Online EKG tutorial: http://education.med.nyu.edu/courses/physiology/course ware/ekg_pt1/ekgmenu.html). The subjects were subsequently randomized into two groups. The students in the control ("classical" instruction) group received no additional instruction, whereas the students in the intervention ("ECGSIM") group received additional instruction using the ECGSIM software (See Table 1). The supplemental instruction using ECGSIM provided students of the ECGSIM group with a three-dimensional concept of the ECG, unlike the two-dimensional concept received by the students of the classical group.

At the end of the preparation phase, each group received instructions for determining the QRS axis. The instructions given to both groups explained the anatomic positions of the different leads, defined the electrical axis, and outlined the steps required for axis calculation. The manual included a sample ECG with all the steps for calculating the axis explained. A unit circle with the different angles was also provided in the manual with the names of the unipolar leads (aVR, -aVR, aVL, and aVF) present. Both groups then received 10 test ECGs, an answer sheet, and a unit circle showing the lead directions and all possible designations for the electrical axis. Each participant was asked to determine the QRS axis. All answers were recorded on an answer sheet on which the students were surveyed for previous experience or education on ECGs.

\section{Electrocardiogram selection}

The ECGs used in the test were chosen to provide an array of different axes and levels of difficulty. ECGs with both normal and abnormal QRS complexes were included in the study. The ECGs were selected from a database of real-patient electrocardiograms, with patient consent of scientific usage from Duke University Hospital. The same 10 ECGs were used when testing both the classical and ECGSIM groups.

\section{Scoring}

All results were scored as correct to the nearest degree, to within a 10-degree range, and to within a 60-degree range to represent poor accuracy in calculating the axis. Each answer that was accurate to the nearest degree was awarded 2 points. Each answer that was accurate within a 10-degree range was awarded 1 point. On the other hand, 1 point was subtracted for every answer that fell between the 10- and 60-degree ranges. This scoring system was used to indicate the level of understanding of the students on the basic concepts of the ECG. As all students were taught to calculate the axis to the nearest degree, the 60degree range was used as a catch-all group to identify students who did not sufficiently understand ECG concepts, or who made a guess at the correct answer. Thus, the study could correctly categorize all students. The investigators serving as the gold standard against which the participants were scored determined the correct QRS mean electrical axis to the nearest degree using the same method taught to the participants. The ECGs were scored by one of the investigators (TAP) in a blinded fashion.

Statistical methods

The student $t$ test was used to determine the significance of the mean percentage of correctly calculated QRS axes in the classical and ECGSIM groups. Results are given as mean values and standard deviations (in parentheses). A $p$ value of $<0.05$ was considered significant.

\section{Results}

\subsection{ECGSIM improved accuracy in calculating mean QRS electrical axis to nearest degree}

Using the scoring system described above, performance on each of the 10 ECGs were determined and averaged for each group. While $6.4 \%( \pm 3.6 \%)$ of those in the control group correctly calculated the mean QRS electrical axis to the nearest degree, $19.5 \%( \pm 4.2 \%)$ of the participants in the intervention group were able to do so $(p=0.002)$ (Figure 1a). Furthermore, on each ECG, the participants in the intervention group performed better than those in the control group, as shown in Figure 3a.

\subsection{ECGSIM improved accuracy in calculating mean QRS electrical axis within 10 degrees}

The same scoring scheme used above was employed in identifying participants that were accurate within 10 degrees of the precise QRS mean axis. As shown in Figure $1 \mathrm{~b}, 21.4 \%( \pm 8 \%)$ of the students in the control group and $45.7 \%( \pm 7.6 \%)$ of the students in the intervention group were able to calculate the mean QRS electrical axis to within 10 degrees $(p=0.044)$. However, 
the impact of ECGSIM was less pronounced as the performance on each ECG varied between which group performed better. This is shown in Figure $3 b$.

\section{3. $\quad$ Reduced diagnostic inaccuracy}

Next we examined which participants missed the 10degree range of accuracy and fell within 60 degrees of the accurate mean QRS axis, a measure of poor ability to calculate the mean QRS axis. In this analysis, $62.9 \%$ $( \pm 7.7 \%)$ of the control group and $47.1 \%( \pm 7 \%)$ of the intervention group fell within this range $(\mathrm{p}=0.001)$.

\section{Tables and figures}

Table 1. Flowchart and Experiment Design

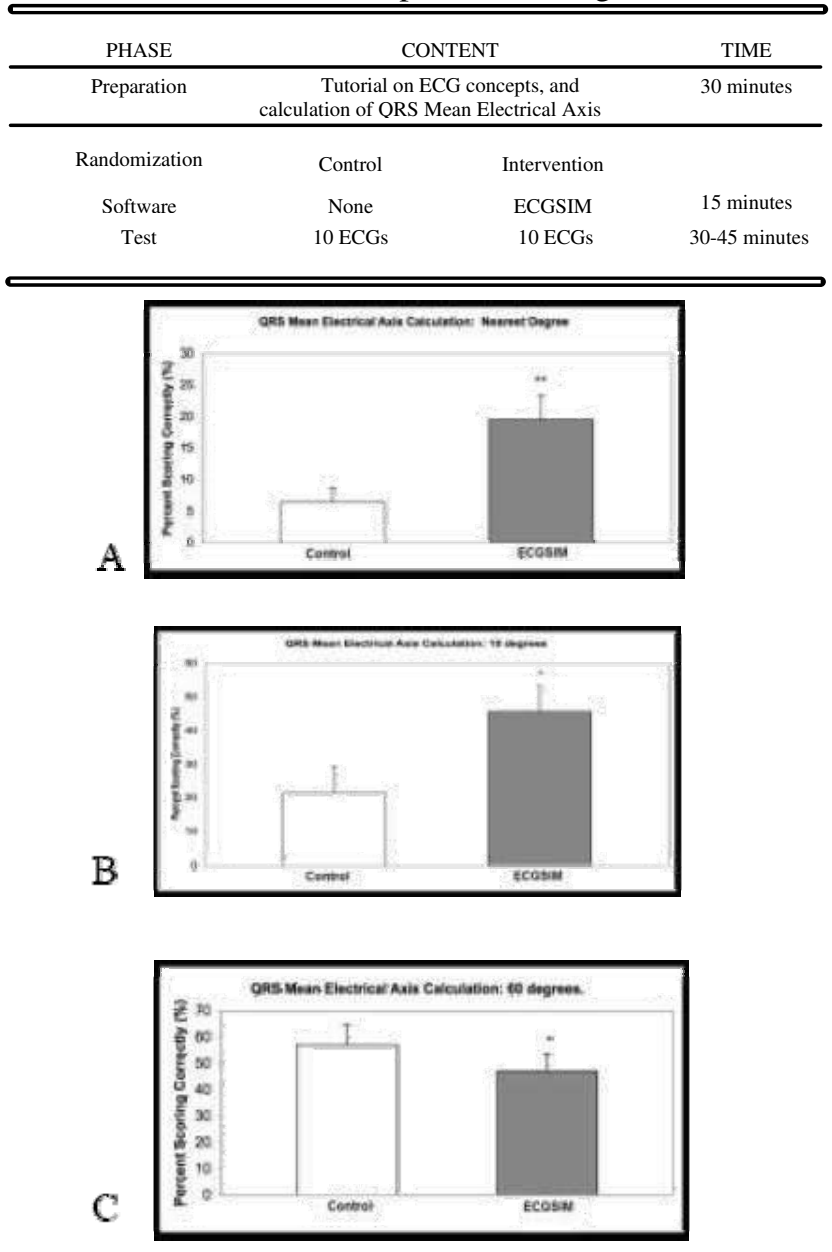

Figure 1. Results from study showing the percentage of participants that correctly calculated the given ECGs to A) the nearest degree, B) within 10 degrees, C) within 60 degrees of correctness. ${ }^{*} \mathrm{p}<0.05,{ }^{* *} \mathrm{p}<0.01$

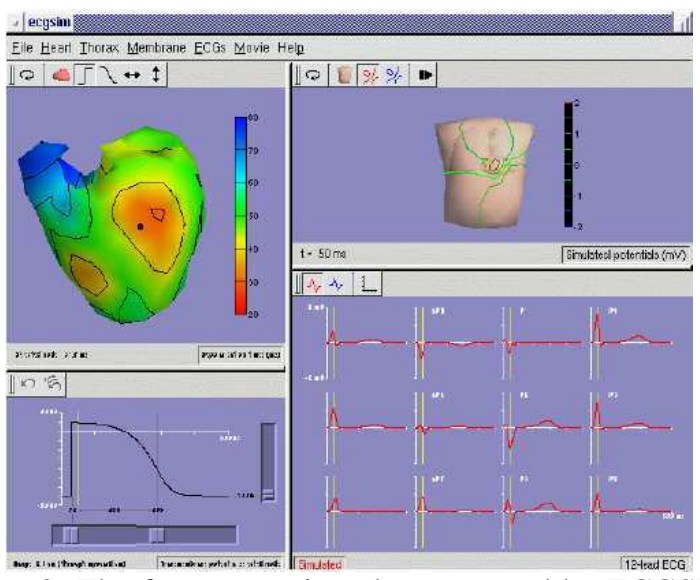

Figure 2. The four pane view demonstrated by ECGSIM. This is a representative image from the ECGSIM program, as used in our study.

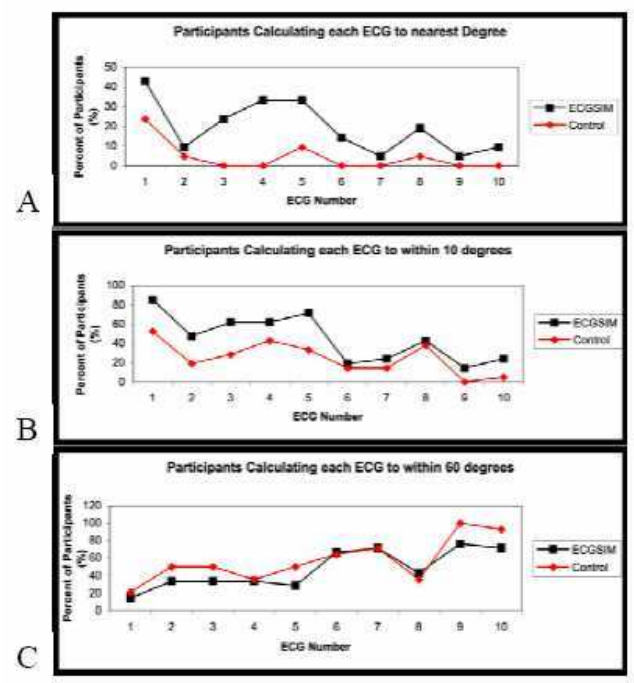

Figure 3. Display of performance by group on each ECG. The percentage is shown for participants scoring to A) the nearest degree, B)within 10 degrees, C)within 60 degrees.

\section{Discussion}

There are several ways of teaching students the basics of the electrocardiogram, and it is not necessarily clear which method produces the best results. Currently, medical schools typically teach students using what this study has referred to as the "classical" method. This approach involves teaching students in a small group- or lecture-based setting, usually with the aid of books, handouts, and/or figures. This method is usually supplemented by exposure to many ECGs during clinical years, as well as one-on-one or case-by-case didactics. It may be difficult for students being exposed to electrocardiography to make a link between these two- 
dimensional approaches and real-time three-dimensional activity actually occurring in the heart that generates the electrocardiographic tracing. ECGSIM is an interactive software, ${ }^{[3,4]}$ that can serve as a supplement to the classical method of teaching ECG interpretation. Learning electrocardiography via ECGSIM offers many advantages. ECGSIM provides students the chance to experience the heart's activities in an interactive setting. More importantly, the software provides the opportunity to study cardiac physiology in three dimensions in an interactive setting, where students can observe the effects of cardiac pathology and lead placement on ECG traces.

Although, it is intuitive that computerized programs can aid in teaching many medical concepts, there have been no studies to document the benefits of softwarebased learning on ECG interpretation. To our knowledge, this is the first study to provide scientific support for ECGSIM that demonstrates the impact of ECGSIM in a direct quantitative comparison with classical methods of teaching ECG concepts. The results of our study indicate that ECGSIM, when used in conjunction with the classical method, gave students a more solid grasp of the principles underlying the ECG, thus giving them an edge in the calculations of important parameters in ECG interpretation. In particular, as shown in Figure 3, when it came to calculating the mean QRS axis most precisely, students taught using ECGSIM in addition to other aids far outperformed their counterparts who had no exposure to ECGSIM. When the study focused on the calculation of the mean QRS axis within 10 degrees, a clinically relevant range, the use of ECGSIM provided a greater degree of accuracy compared with not using the software. However, the difference between the two groups in this particular parameter was not as robust as the difference observed in the groups' ability to calculate the mean QRS axis to the nearest degree, suggesting that the utility of ECGSIM lies mostly in providing a higher level of understanding. Lastly, the fact that students using ECGSIM were far less likely to obtain axis values in the 10 to 60-degree range, a measure of poor understanding, further supports the interpretation that use of the software gave students an improved understanding of the ECG.

Some potential drawbacks to our study include the fact that students in the ECGSIM group received an extra 15 minutes of didactics compared to the participants who had no exposure to ECGSIM. We argue that time is unlikely to account for the differences observed since the extra time spent, was spent using ECGSIM to explain the same concepts that were previously introduced during the classical method portion. Further, the same argument could be raised if the two groups spent the same amount of time being taught, the control group would need to receive more didactics than the ECGSIM group. Another potential criticism is the fact that students spent only 30 minutes being introduced to the concepts of the ECG, a relatively short time when the extensive field of electrocardiography is concerned. A short, focused, and solid background was chosen in this group so as not to overwhelm the students. The participants were previously unexposed to the ECG, and therefore a broad, far reaching teaching session would likely have complicated the session. Further, the study chose to use this group because the impact of ECGSIM would be less significant in a population of participants with previous knowledge of the electrocardiogram and would not truly reflect the ability of this software to improve the understanding of the concepts of electrocardiography.

\section{Conclusion}

In conclusion, students whose instruction in ECG interpretation is supplemented with the ECGSIM software demonstrate greater accuracy in calculating the mean QRS axis than those who are instructed solely by the classical approach. Based on the findings of this study, the utility of the ECGSIM software in medical school would be of great value. It is intuitive based on this study to include more interactive programs to help simplify many, often-complicated, medical concepts. However, it is possible that not all interactive computer programs would show the same benefit as ECGSIM, and some may in fact be detrimental. For this reason, studies such as this should be undertaken to show the benefits of implementing computer programs in medical education.

\section{Acknowledgements}

Special thanks to Dr. Adriaan van Oosterom, Michiya Nishino, Kathy Shuping and Beverly Perkins.

\section{References}

[1] Pahlm US, O'Brien JE, Pettersson J, Pahlm O, White T, Maynard C, Wagner GS. Comparison of teaching the basic electrocardiographic concept of frontal plane QRS axis using the classical versus the orderly electrocardiogram limb lead displays. American Heart Journal 1997;134:1014-18.

[2] Hooft van Huysduynen B, et al. Dispersion of repolarization in cardiac resynchronization therapy. Heart Rhythm 2005; 2;1286-1293.

[3] Hooft van Huysduynen B, et al. Validation of ECG indices of ventricular repolarization heterogeneity. J Cardiovasc Electrophysiol 2005; 16;1097-1103.

[4] van Oosterom A, Oostendorp TF, ECGSIM an Interactive Tool for Simulating QRST Waveforms. Heart 2004; 165168

Address for correspondence:

Timothy A. Patuwo

2517 Wordsworth Street, Houston, Texas 77030, USA 